Classification

Physics Abstracts

$06.50-07.80-82.80$

\title{
Automated processing of parallel-detection EELS data
}

\author{
Michael K. Kundmann(*) and Ondrej L. Krivanek \\ Gatan R\&D, 6678 Owens Drive, Pleasanton, CA 94588, U.S.A.
}

(Received February 18, 1991; accepted April 09, 1991)

\begin{abstract}
An algorithm for automatic detection and identification of edges in an EELS spectrum is presented. It has the following features: 1) it compresses the dynamic range of EELS spectra and enhances the ionization edge signals via difference transforms, 2) it removes residual background, thereby isolating sharp features associated with the edge thresholds and noise, 3 ) it distinguishes true edge-threshold features from noise via statistical analysis. In addition to paving the way for rapid, automated EELS elemental analysis, the algorithm is capable of detecting edges which are easily overlooked by human analysts.
\end{abstract}

\section{Introduction.}

We would like to be able to carry out EELS analysis of materials as rapidly as possible. Due to parallel detection EELS we can now acquire spectra very quickly, but analysis of the spectra to get the chemical composition still takes time, because it requires input from the analyst. If spectrum analysis could be automated and placed completely under computer control, the total time for EELS analysis would be reduced considerably.

Deciding which features are present in a spectrum and which elements are worth analyzing for is an important first step in EELS data analysis. Once this has been established, most EELS analysis software can take over, carrying out the numerical computations and returning the sample's chemical composition. Until now, however, the initial step of edge identification has been left to the analyst.

We have developed an algorithm which can locate and identify spectrum features without input from the analyst.

\section{The edge-detection algorithm.}

An edge-detection algorithm must do two things. It must first locate candidate edge thresholds, i.e. those points where an abrupt change in count rate occurs. Then it must distinguish the true edge thresholds from count rate changes due to near-edge fine structure, noise, and detector artifacts. For the human analyst, it is easy to see where "abrupt changes" in the spectrum occur,

$\left({ }^{*}\right)$ Current address: 189A Wildwood Avenue, Worcester, MA 01603, U.S.A. 
but a computer algorithm requires a precise numerical definition of what constitutes an "abrupt change". The problem is that EELS edge shapes and jump ratios vary greatly, as does the overall spectrum count level, due to the underlying inverse power-law dependence. It is difficult, within the context of the raw EELS spectrum, to formulate universal numerical criteria which characterize all edge thresholds. Our algorithm solves these problems in three steps (Fig. 1):

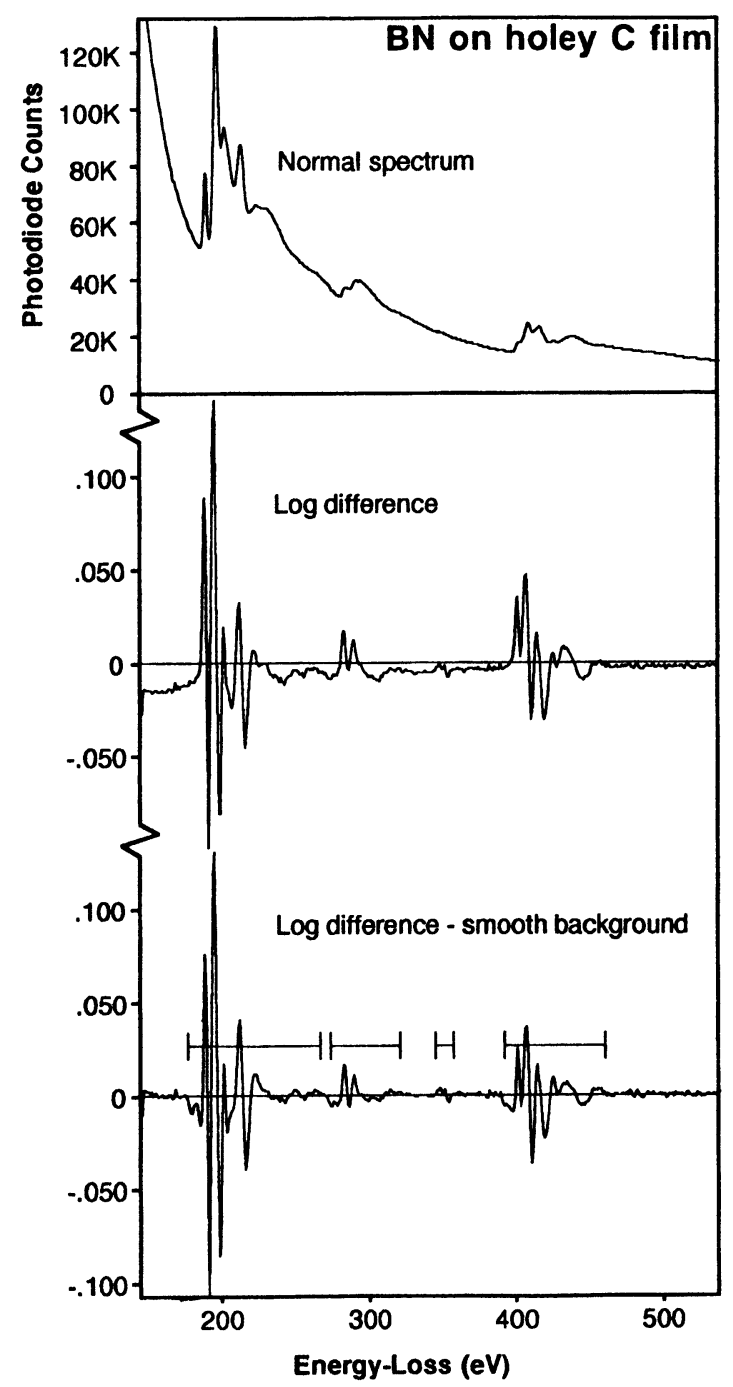

Fig. 1. - Illustration of each step of the automatic edge detection algorithm. Bars on lower plot indicate energy-loss ranges identified as containing significant edge signals.

1) A difference transform of the spectrum is computed [1-4]. This accentuates the edge thresholds over the slowly varying background and reduces the dynamic range of the spectrum. In addition, the steps and slope changes at edge thresholds in the raw spectrum are thereby converted 
to pronounced peaks, making it easier to specify numerical criteria which characterize the thresholds.

2) Any remaining background is removed by applying a strong smoothing filter to the transformed spectrum and subtracting the result from same. This leaves only the signals of the edge thresholds and their associated fine structure riding on symmetrically distributed bipolar noise.

3) The last step determines which signals stand out above the noise and attempts to group those associated with the same ionization edges. The energy thresholds of the detected signals are then used to identify the atomic shells to which they correspond.

2.1 DIFFERENCE TRANSFORM. - Numerous variations on difference transforms are possible. We have tried the following four in the edge detection algorithm: first difference, second difference, log difference, and one we have called the " $R$-plot." The first, second, and log difference transforms are discrete analogs of the spectrum's first, second, and logarithmic derivatives, respectively, with respect to energy loss. The $R$-plot represents the slope of the spectrum as plotted on a log-log scale. It is analogous to the logarithmic derivative with respect to the log of the energy loss. The inspiration for the $R$-plot is that it should give a constant value in edge-free background regions of the spectrum due to their approximate power-law dependence.

Given a discrete EELS spectrum, $S(i), i=$ channel number, the precise mathematical definitions of these transforms are as follows:

1) First difference, $\Delta_{1}\{S(i)\}$, based on $\mathrm{d} S / \mathrm{d} E$ :

$$
\Delta_{1}\{S(i)\}=S(i+\Delta i)-S(i-\Delta i)
$$

2) Second difference, $\Delta_{2}\{S(i)\}$, based on $\mathrm{d}^{2} S / \mathrm{d} E^{2}$ :

$$
\Delta_{2}\{S(i)\}=2 S(i)-S(i+\Delta i)-S(i-\Delta i)
$$

3) Log difference, $\Delta_{\mathrm{L}}\{S(i)\}$, based on $\mathrm{d}(\ln S) / \mathrm{d} E=(1 / S) \mathrm{d} S / \mathrm{d} E$ :

$$
\Delta_{\mathrm{L}}\{S(i)\}=[S(i+\Delta i)-S(i-\Delta i)] / S(i)
$$

4) $R$-plot, $\Delta_{R \mathrm{P}}\{S(i)\}$, based on $\mathrm{d}(\ln S) / \mathrm{d}(\ln E)=(E / S) \mathrm{d} S / \mathrm{d} E$ :

$$
\Delta_{R \mathrm{P}}\{S(i)\}=E(i)[S(i+\Delta i)-S(i-\Delta i)] / S(i)
$$

In the above definitions, $\Delta i$ represents a channels offset and $E(i)$ gives the energy loss associated with channel $i$. Figure 2 illustrates each of the above four transforms applied to the sample spectrum of figure 1.

2.2 REMOVAL OF RESIDUAL BACKGROUND. - In order that a simple threshold criterion suffice for distinguishing candidate edges from noise, it is necessary that the transformed spectrum have a constant baseline. Although all the transforms flatten the background considerably, none yields a constant baseline. By passing the transformed spectrum through a strong smoothing filter and subtracting the result from the original, a zero baseline is obtained. Mathematically, this zero baseline transform, $Z B\{\}$, is defined as

$$
Z B\left\{S^{\mathrm{t}}(i)\right\}=S^{\mathrm{t}}(i)-S M\left\{S^{\mathbf{t}}(i)\right\}
$$

where $S M\{\}$ is the strong smoothing filter and $S^{\mathrm{t}}(i)=\Delta_{x}\{S(i)\}$. Numerous implementations of smoothing filters exist and the precise definition of $S M\{\}$ is probably not critical to the performance of the algorithm as long as $S M\{\}$ smooths out most of the edge threshold structure. We use a top hat filter, which replaces each spectrum channel with the average of $n$ neighboring channels. Typically, we choose $n=41$. 

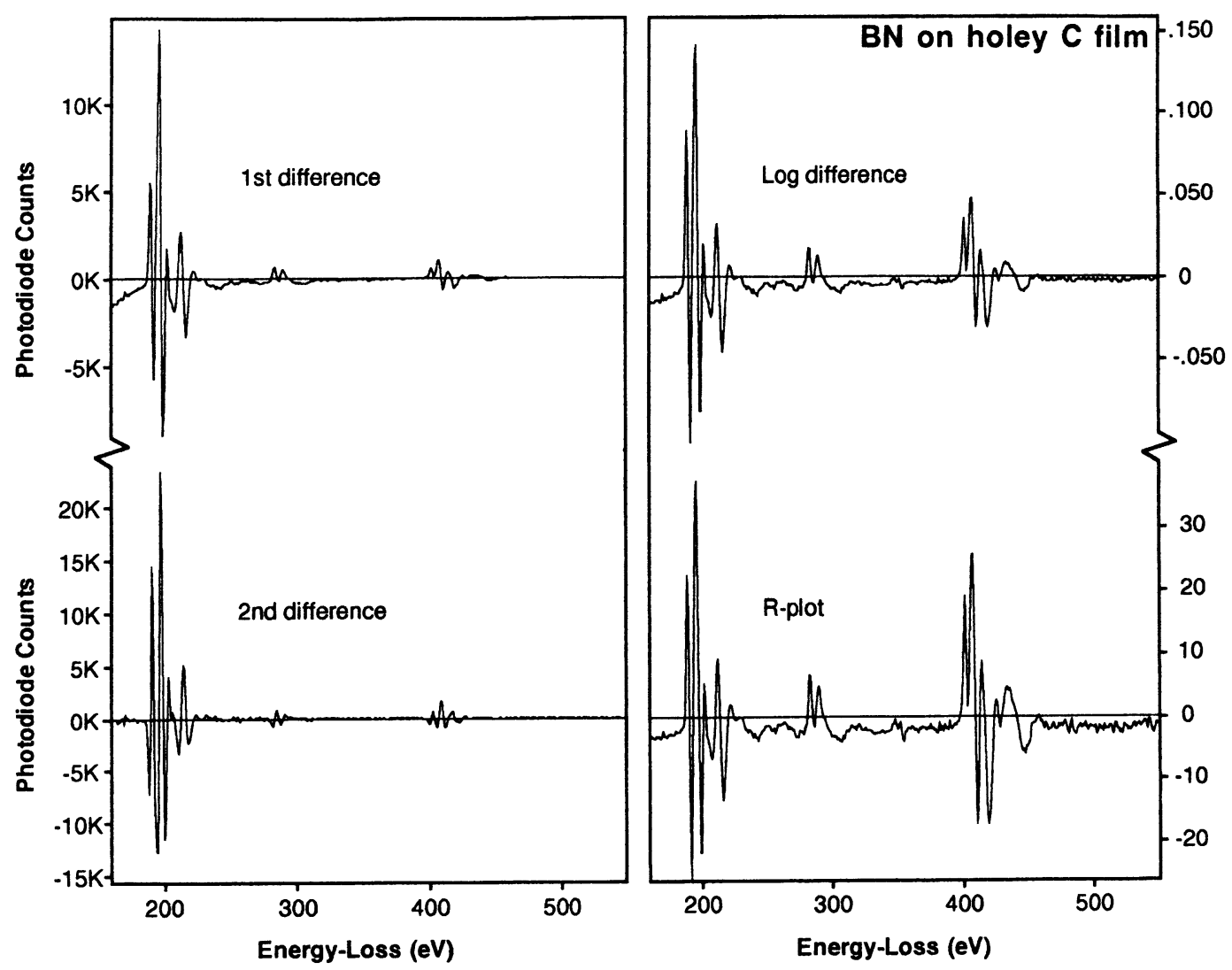

Fig. 2. - First, second, and logarithmic difference and $R$-plot transforms of the $\mathrm{BN}$ on $\mathrm{C}$ spectrum of figure 1. To reduce noise amplification, spectrum was smoothed with a 3-channel top-hat filter before transformation. For each transform, $\Delta i=3$ channels.

2.3 IDENTIFICATION OF SIGNIFICANT FEATURES. - The final step is to formulate criteria which distinguish edge-related features from noise, and edge thresholds from near-edge fine structure. For both of these purposes, we have found it useful to analyze the unipolar segments of the transformed spectrum. In this way, both the intensity and non-random distribution of edge-related features in the spectrum transform are used to distinguish them from the weak and randomly distributed noise (Fig. 3). Specifically, a histogram of the unipolar segment intensity is collected. Noise segments have low integrated intensity and cluster in a peak centered on zero. True edge feature segments appear in the tails of the distribution. Assuming the segment intensities in the edge-free regions of the spectrum are randomly distributed, a reasonable cutoff for separating edge features from noise is a $3 \sigma$ criterion, where $\sigma$ is the standard deviation of the distribution excluding edge features. In order to determine $\sigma$, an iterative approach is used. First, the standard deviation is computed using all points in the histogram. All points more than $3 \sigma$ from zero are taken out and the standard deviation is recomputed. This process is repeated until all retained points lie within $3 \sigma$ of zero. The unipolar segments associated with the excluded points are marked as true edge features.

To locate edge thresholds, we group all contiguous marked segments into a single edge. Overlapping edges are resolved by using the fact that edge threshold features tend to be the narrowest 

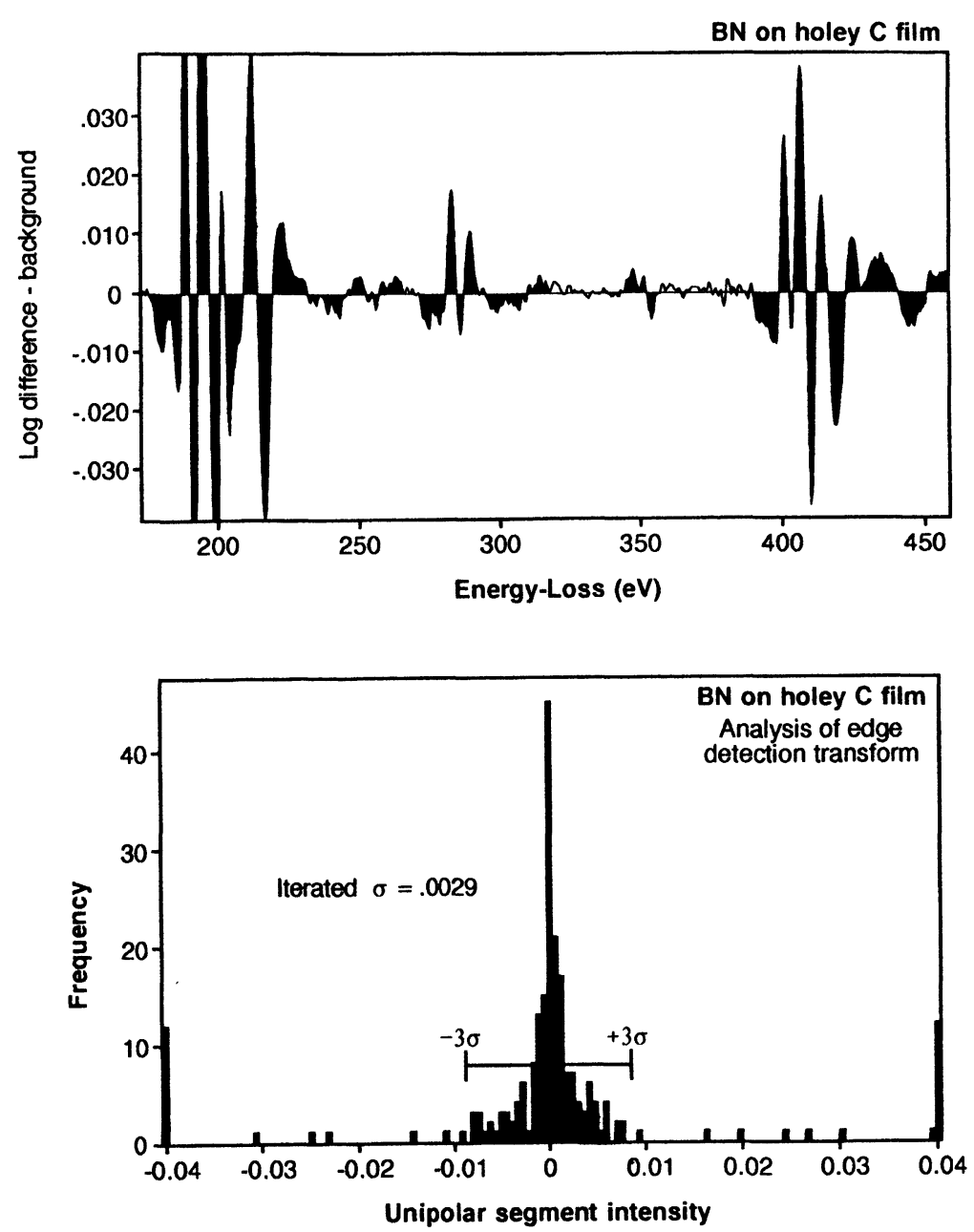

Fig. 3. - Top: Detailed view of edge-detection tranform based on log difference for spectrum of figure 1 . Note that it may be viewed as a collection of unipolar segments. Segments associated with edge features are sharply peaked and of high integrated intensity. Darkened segments are those deemed to be above noise based on statistical analysis of segment intensity distribution. Bottom: Histogram of unipolar segment intensities. Horizontal bar indicates iteratively calculated $\sigma$ using a $3 \sigma$ cutoff criterion.

and the most intense. A parameter which reflect this information is the segment intensity divided by its width in channels. This parameter tends to be very large at edge thresholds and falls off rapidly thereafter. If it undergoes an abrupt increase within a marked set of segments, the corresponding segment is taken to mark the threshold of another edge.

Once all distinct edge signals have been marked, the first peak (for $\Delta_{1}, \Delta_{\mathrm{L}}$, and $\Delta_{R \mathrm{P}}$ ) or the zero immediately preceding the first peak (for $\Delta_{2}$ ) in each marked region is taken as the edge threshold (Fig. 4). By comparing the measured edge threshold energies with tabulated [5, 6] ionization energy data, the identity of each marked edge is established. In the example of figure 1, edge thresholds were located at $188.5,282.4,347.1$, and $400.5 \mathrm{eV}$, indicating the presence of B K, $\mathrm{C} \mathrm{K}, \mathrm{Ca} \mathrm{L}_{3,2}$, and $\mathrm{N} \mathrm{K}$ edges. 


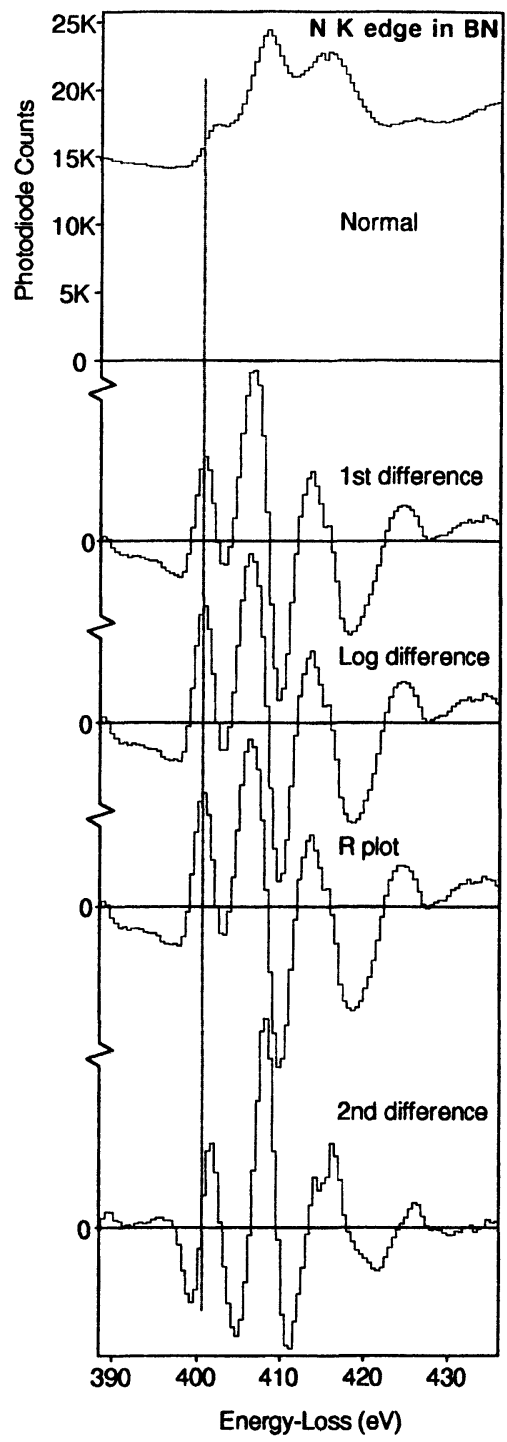

Fig. 4. - Relationship between an edge threshold and peaks (or zeros) of its corresponding edge-detection tranform. Results based on various difference transforms are shown.

\section{Examples.}

Figures 5a,b,c,d demonstrate the effectiveness of the algorithm in locating and identifying the edges in a number of typical EELS spectra. Some of the strengths and weaknesses of the algorithm are illustrated.

In figure 5a, four edges with varying degrees of overlap appear in the spectrum. The edge detection transform gives a very clear signal for each edge. Although the significant structure of the $\mathrm{O} \mathrm{K}$ edge runs on into the $\mathrm{Cr} \mathrm{L}$ edge, the algorithm applies the peak/width abrupt change criterion to successfully resolve these two edges. Figure $5 \mathrm{~b}$ shows that even fairly weak edges, 


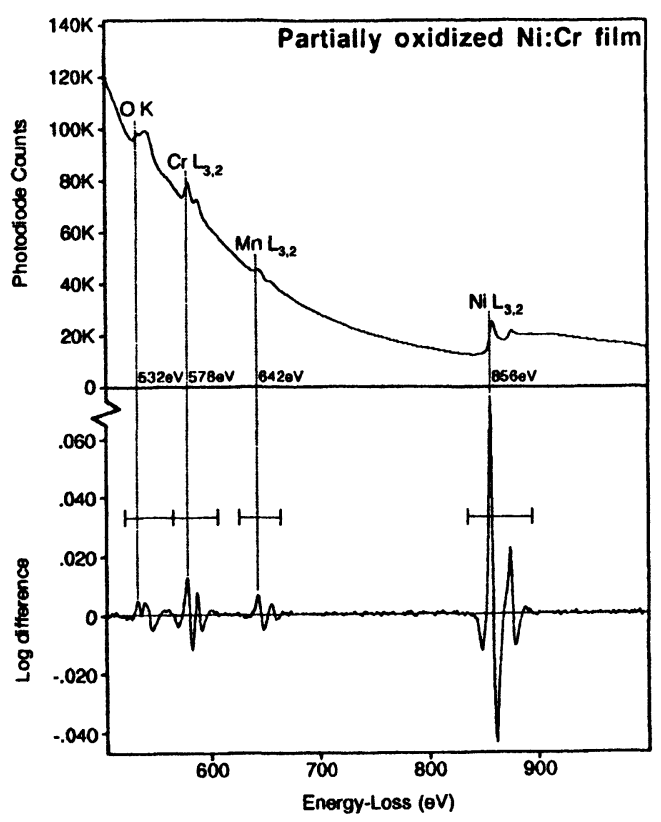

a)

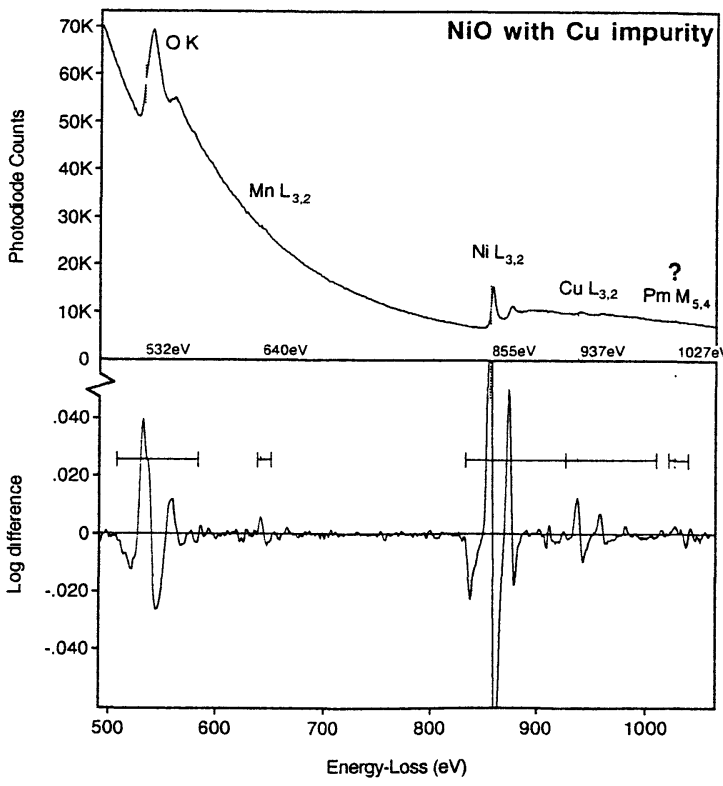

c)

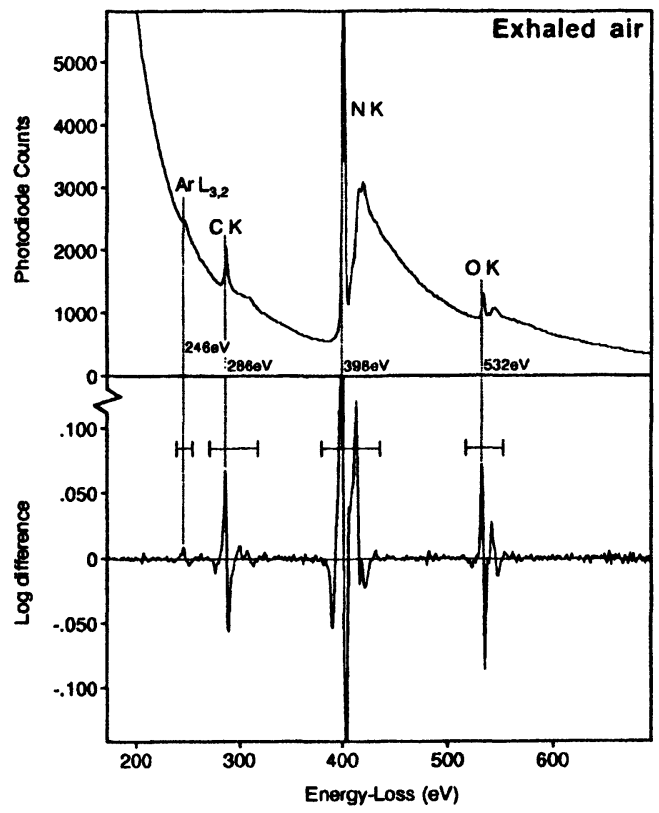

b)

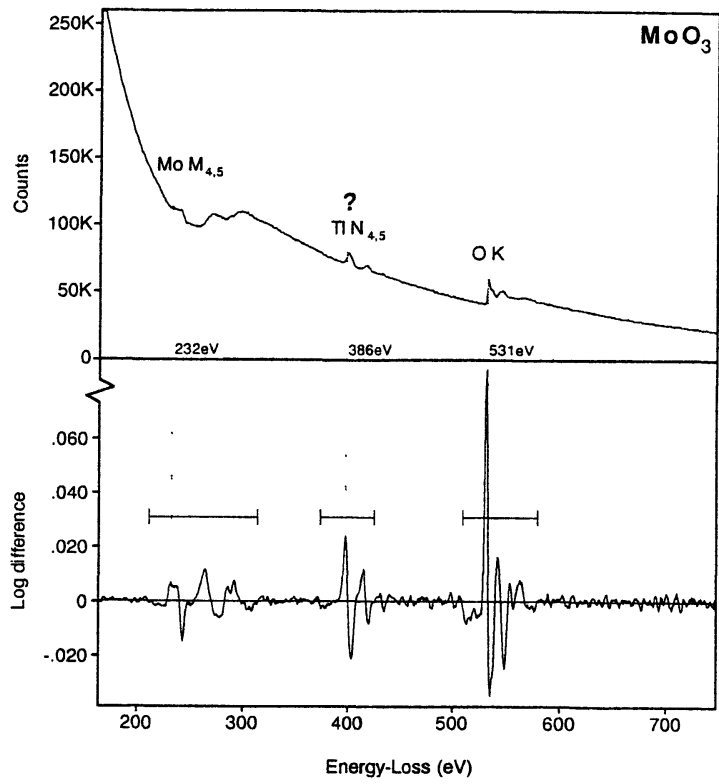

d)

Fig. 5. - Results of the automated edge-detection algorithm on several typical EELS spectra: a) parallel EELS of evaporated transition metal film, partially oxidized by heating in air, b) parallel EELS of exhaled air sample in an environmental cell, c) parallel EELS of a NiO film, and d) serial EELS of a molybdenum trioxide particle taken from reference [5]. 
such as the Ar edge measured from an air sample, can be detected and correctly identified by the algorithm. This sensitivity is confirmed by the results shown in figure $5 \mathrm{c}$, in which barely discernible $\mathrm{Cu}$ and $\mathrm{Mn}$ edges are easily picked out by edge-detection routine.

One of the problems with the algorithm also comes up in figure 5c. Simply comparing a detected edge threshold energy with tabulated values is not a completely reliable way to identify an edge. Edge thresholds can shift slightly due to chemical valence and coordination effects and do not always occur at the tabulated values. It is highly unlikely that the sample in $5 \mathrm{c}$ contained significant amounts of Pm (promethium), a naturally radioactive element. A manual search of the edge energy table yields more likely candidates for the feature detected at $1027 \mathrm{eV}$, either $\mathrm{Zn}$ $\mathrm{L}_{3}(1020 \mathrm{eV})$ or $\mathrm{Ni} \mathrm{L}_{1}(1008 \mathrm{eV})$. One obvious improvement to the edge identification algorithm would be to first check for companion edges to the ones already identified (such as the $\mathrm{Ni} \mathrm{L}_{1}$ ).

The example of figure 5d shows that the algorithm still works in cases where the edge threshold is characterized by a change in slope rather than by a pronounced peak. Note that the $\mathbf{M o ~}_{4,5}$ edge is properly detected and identified despite the "false" edge which appears at about $255 \mathrm{eV}$ due to its delayed maximum. However, another edge is misidentified in this example as well. The feature at $386 \mathrm{eV}$ identified as $\mathrm{Tl} \mathrm{N}_{4,5}$ is actually the $\mathrm{Mo} \mathrm{M}_{3,2}$ edge which has a tabulated threshold energy of $392 \mathrm{eV}$.

In order to avoid trying to make a complete chemical analysis following a mistaken edge identification in cases such as the one above, the program includes an extra step in which the analyst is informed of each identification made, and of other candidate edges in the vicinity of the detected feature.

\section{Discussion.}

The ultimate sensitivity of the edge-detection algorithm is largely determined by the signal-tonoise ratio achieved in the difference transform of the original spectrum. Both detector artifacts and the type of difference transform used have significant influence on the signal-to-noise ratio.

4.1 AVOIDING DETECTOR ARTIFACTS. - The two most important sources of detector artifacts in a parallel-detection EELS system are channel-to-channel gain variation and any fixed-pattern detector background. Instead of measuring $S(i)$, one actually measures $S^{\mathrm{m}}(i)$, given by

$$
S^{\mathbf{m}}(i)=g(i) S(i)+b(i)
$$

where $g(i)$ is the relative detector gain and $b(i)$ is the detector background at channel $i$. The effects of these artifacts on the difference transforms is shown by substituting the above into the difference transfrom definitions:

1) First difference:

$$
\begin{aligned}
\Delta_{1}\{S(i)\} & =S(i+\Delta i)-S(i-\Delta i) \\
& =g(i+\Delta i) S(i+\Delta i)+b(i+\Delta i)-g(i-\Delta i) S(i-\Delta i)-b(i-\Delta i) \\
& =[g(i+\Delta i) S(i+\Delta i)-g(i-\Delta i) S(i-\Delta i)]+[b(i+\Delta i)-b(i-\Delta i)]
\end{aligned}
$$

2) Second difference:

$$
\begin{aligned}
\Delta_{2}\{S(i)\}= & 2 S(i)-S(i+\Delta i)-S(i-\Delta i) \\
= & 2 g(i) S(i)+2 b(i+\Delta i)-g(i+\Delta i) S(i+\Delta i)+b(i+\Delta i) \\
& \quad-g(i-\Delta i) S(i-\Delta i)-b(i-\Delta i) \\
= & {[2 g(i) S(i)-g(i+\Delta i) S(i+\Delta i)-g(i-\Delta i) S(i-\Delta i)] } \\
& \quad+[2 b(i)-b(i+\Delta i)-b(i-\Delta i)]
\end{aligned}
$$


3) Log difference:

$$
\begin{aligned}
\Delta_{\mathrm{L}}\{S(i)\} & =[S(i+\Delta i)-S(i-\Delta i)] / S(i) \\
& =\frac{g(i+\Delta i) S(i+\Delta i)+b(i+\Delta i)-g(i-\Delta i) S(i-\Delta i)-b(i-\Delta i)}{g(i) S(i)+b(i)}
\end{aligned}
$$

4) $R$-plot:

$$
\begin{aligned}
\Delta_{R \mathrm{P}}\{S(i)\} & =E(i)[S(i+\Delta i)-S(i-\Delta i)] / S(i) \\
& =\frac{E(i)[g(i+\Delta i) S(i+\Delta i)+b(i+\Delta i)-g(i-\Delta i) S(i-\Delta i)-b(i-\Delta i)]}{g(i) S(i)+b(i)}
\end{aligned}
$$

In all cases, the detector artifacts propagate through the difference transform and obscure the true difference spectrum. Because differences are taken, the artifact contribution usually increases relative to the true signal, tending to nullify some of the benefits of the difference transform.

A method for bypassing these difficulties with difference transforms is available, as first described by Shuman and coworkers [1]. To avoid artifact effects, one can use independent, energyshifted, spectrum readouts in place of $S(i-\Delta i)$ and $S(i+\Delta i)$, i.e.

$$
\begin{aligned}
& S(i-\Delta i) \rightarrow S^{\mathrm{m}}{ }_{-}(i)=g(i) S_{-}(i)+b(i) \\
& S(i+\Delta i) \rightarrow S^{\mathrm{m}}{ }_{+}(i)=g(i) S_{+}(i)+b(i)
\end{aligned}
$$

where $S^{\mathrm{m}}-(i)\left(S^{\mathrm{m}}+(i)\right)$ is measured by physically shifting the spectrum to lower (higher) energies (e.g. via an applied spectrometer drift tube voltage) relative to a given channel of the detector array. Substituting the above into the difference transform definitions, the following are obtained:

1) First difference:

$$
\begin{aligned}
\Delta_{1}\{S(i)\} & =S^{\mathrm{m}}{ }_{+}(i)-S^{\mathrm{m}}-(i) \\
& =g(i)\left[S_{+}(i)-S_{-}(i)\right]
\end{aligned}
$$

2) Second difference:

$$
\begin{aligned}
\Delta_{2}\{S(i)\} & =2 S^{\mathrm{m}}(i)-S^{\mathrm{m}}+(i)-S^{\mathrm{m}}{ }_{-}(i) \\
& =g(i)\left[2 S(i)-S_{+}(i)-S_{-}(i)\right]
\end{aligned}
$$

3) Log difference:

$$
\begin{aligned}
\Delta_{\mathrm{L}}\{S(i)\} & =\left[S^{\mathrm{m}}+(i)-S^{\mathrm{m}}-(i)\right] / S^{\mathrm{m}}(i) \\
& =\left[S_{+}(i)-S_{-}(i)\right] /[S(i)+b(i) / g(i)]
\end{aligned}
$$

4) $R$-plot:

$$
\begin{aligned}
\Delta_{R \mathrm{P}}\{S(i)\} & =E(i)\left[S^{\mathrm{m}}+(i)-S^{\mathrm{m}}-(i)\right] / S^{\mathbf{m}}(i) \\
& =E(i)\left[S_{+}(i)-S_{-}(i)\right] /[S(i)+b(i) / g(i)]
\end{aligned}
$$

With this approach, the fixed pattern background drops out completely in the 1st and 2nd difference transforms. Furthermore, the channel-to-channel gain variation is not amplified in these cases since it factors out as a common multiplier. For the log difference and $R$-plot transforms, the channel-to-channel gain variation actually drops out completely except for a small effect due to the detector background of the unshifted component spectrum in the denominator. If the detector background is first calibrated and subtracted, then all detector artifacts will be completely absent from the log difference and $R$-plot transforms.

The higher signal-to-noise achieved through the use of measured energy-shifted spectrum readouts and the consequent improved edge-detection sensitivity of the algorithm is illustrated in figure 6. When the numerically computed edge-detection transform is used, the very weak Mn L edge is missed entirely and two detector artifacts are mistaken for real spectrum features. These errors are completely avoided by using energy-shifted readouts to form the edge-detection transform. 


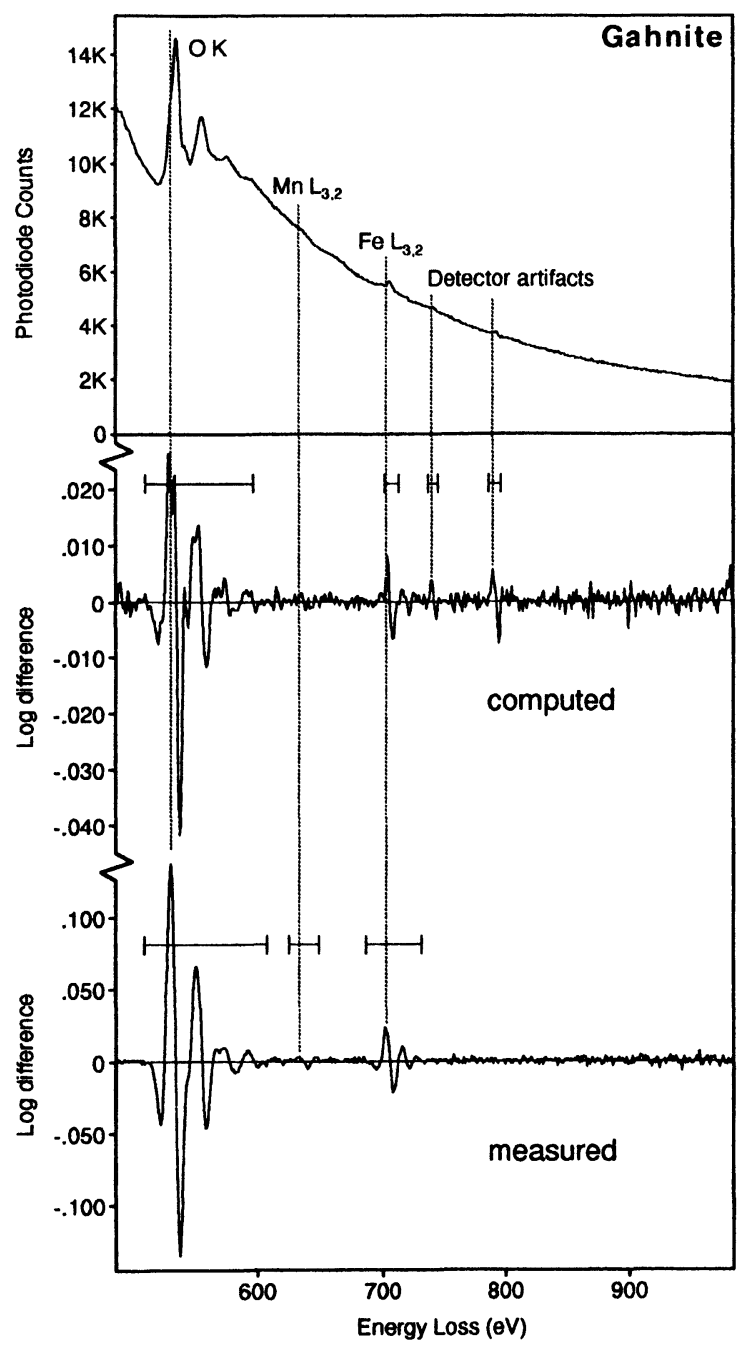

Fig. 6. - Comparison of numerically computed edge-detection transform with that obtained by measuring and combining several energy-shifted spectrum readouts for the Gahnite spectrum shown at top.

4.2 CHOICE OF DIFFERENCE TRANSFORM. - In principle, the edge detection algorithm could be based on any of the difference transforms discussed above. In practice, because each transform has a different effect on the signal to noise ratio and on the energy-dependence of the noise, they are not all equally effective.

In order to make quantitative comparisons between the difference transforms, we applied both the computed and measured versions of each difference transform to the Gahnite data and measured the following quantities for each: 1) the RMS noise per channel in each of four signal-free energy loss regimes, $475-505 \mathrm{eV}, 660-690 \mathrm{eV}, 750-780 \mathrm{eV}$, and $950-980 \mathrm{eV}$, and 2) the signal-to-noise ratios at the oxygen $\mathrm{K}$ and iron L edges. The latter were determined as follows: 1) the signal at each edge was taken as the sum of the absolute values of the difference transform counts over the energy range highlighted by the edge-detection algorithm, 2) an estimate of the RMS noise per channel within the highlighted range was interpolated from the RMS noise measurements, 3) the 
noise at each edge was taken as the estimated RMS noise per channel times the square root of the number of channels within the highlighted range. The results are summarized in tables I and II.

Table I. - Signal-to-noise ratios achieved in edge-detection transforms based on numerically computed difference spectra of gahnite sample.

\begin{tabular}{|l|rrll|}
\hline & \multicolumn{5}{|c|}{ Difference transform type } \\
& \multicolumn{1}{|c|}{ 1 st } & 2 nd & Log & $R$-plot \\
\hline RMS noise/ch $(475-505 \mathrm{eV})$ & 18.32 & 39.2 & $1.550 \times 10^{-3}$ & 0.505 \\
RMS noise/ch $(660-690 \mathrm{eV})$ & 4.08 & 9.90 & $0.681 \times 10^{-3}$ & 0.306 \\
RMS noise/ch $(750-780 \mathrm{eV})$ & 4.09 & 10.42 & $1.007 \times 10^{-3}$ & 0.511 \\
RMS noise/ch $(950-980 \mathrm{eV})$ & 2.67 & 6.20 & $1.351 \times 10^{-3}$ & 0.866 \\
& & & & \\
O K signal $\quad(510-610 \mathrm{eV})$ & 13550 & 13670 & 1.17 & 422 \\
O K noise & 158 & 346 & 0.0157 & 5.72 \\
O K $S / N$ & 85.7 & 39.5 & 74.2 & 73.9 \\
& & & & \\
Fe L signal $\quad(690-730 \mathrm{eV})$ & 751 & 1120 & 0.1404 & 66.1 \\
Fe L noise & 36.5 & 90.9 & 0.00755 & 3.66 \\
Fe L $S / N$ & 20.6 & 12.3 & 18.6 & 18.1 \\
\hline
\end{tabular}

Table II. - Signal-to-noise ratios achieved in edge-detection transforms based on directly measured difference spectra of gahnite sample.

\begin{tabular}{|l|rrll|}
\hline & \multicolumn{5}{|c|}{ Difference transform type } \\
& 1 st & 2 nd & Log & $R$-plot \\
\hline RMS noise/ch $(475-505 \mathrm{eV})$ & 16.3 & 24.8 & $0.672 \times 10^{-3}$ & 0.163 \\
RMS noise/ch $(660-690 \mathrm{eV})$ & 14.6 & 23.6 & $1.21 \times 10^{-3}$ & 0.410 \\
RMS noise/ch $(750-780 \mathrm{eV})$ & 11.6 & 19.5 & $1.43 \times 10^{-3}$ & 0.546 \\
RMS noise/ch $(950-980 \mathrm{eV})$ & 10.7 & 16.3 & $2.67 \times 10^{-3}$ & 1.285 \\
& & & & \\
O K signal $\quad(510-610 \mathrm{eV})$ & 117900 & 87030 & 5.09 & 1380 \\
O K noise & 217 & 342 & 0.0133 & 4.04 \\
O K $S / N$ & 542 & 255 & 383 & 341 \\
& & & & \\
Fe L signal $\quad(690-730 \mathrm{eV})$ & 6060 & 5760 & 0.565 & 200 \\
Fe L noise & 117 & 193 & 0.0118 & 4.27 \\
Fe L $S / N$ & 51.8 & 29.9 & 47.9 & 46.8 \\
\hline
\end{tabular}

The edge-detection algorithm, as described above, requires that the RMS noise per channel be fairly constant throughout the spectrum. If it is not, then clearly defined edge signals in a lownoise region of the spectrum might be lost in the higher noise of another region of the spectrum 
when the histogram of segment intensities is compiled and analyzed. Another consideration is that the transform which preserves the highest signal to noise ratio is preferable since this optimizes the edge-detection sensitivity of the algorithm. For numerically computed difference transforms, table I shows that the noise is most nearly constant for the log difference and the $R$-plot, the variation being slightly smaller for the log difference. Within measurement uncertainties, the $S / N$ ratios for these transforms are the same. Thus, the log difference gives the best result when the difference transform must be computed numerically. For measured difference transforms, table II shows that the 1 st and 2 nd differences have the least amount of noise variation. Of the two, the first difference yields a clearly superior $S / N$ ratio. Thus, the first difference gives the best result when the difference transforms can be measured directly. Taken together, the two tables also demonstrate how much better the $S / N$ ratio is for the case of the measured difference than for the numerically computed one.

\section{Conclusions.}

We have developed an algorithm which automatically detects and identifies the edges present in an EELS spectrum. The algorithm requires no input from the analyst and therefore paves the way for rapid, automated analysis of EELS data. The technique achieves its greatest sensitivity when combined with the difference spectrum acquisition capabilities of parallel-detection EELS spectrometers.

\section{References}

[1] Shuman H., KRUIT P. and Somlyo A.P., Microbeam Analysis - 1983 (San Francisco Press, San Francisco, 1983) 247-251.

[2] ShUMAN H. and KRUIT P., Rev. Sci. Inst. 56 (1985) 231.

[3] ZALUZEC N.J., Ultramicroscopy 18 (1985) 185.

[4] ZALUZEC N.J., Analytical Electron Microscopy - 1987 (San Francisco Press, San Francisco, 1987) 275279.

[5] AHN C.C. and KRIVANEK O.L., EELS Atlas, (Gatan, Inc. Warrendale, PA, 1983).

[6] BEARDEn J.A. and BURR A.F., Rev. Mod. Phys. 39 (1967) 125. 